\title{
Comparing Free-Free and Shaker Table Model Correlation Methods Using Jim Beam
}

\author{
James Ristow, NASA Kennedy Space Center \\ Kenneth Wayne Smith Jr., Georgia Institute of Technology \\ Nathaniel Johnson, University of Oklahoma \\ Jackson Kinney, University of Alabama Huntsville
}

\begin{abstract}
Finite element model correlation as part of a spacecraft program has always been a challenge. For any NASA spacecraft, a Coupled Loads Analysis (CLA) is used to predict the coupled system response of the spacecraft and launch vehicle. The accuracy of the CLA is highly dependent on the precision of the frequencies and mode shapes extracted from the spacecraft model. NASA standards require the spacecraft model used in the final Verification Loads Cycle to be correlated by a modal test. Due to budgetary and time constraints, most programs opt to correlate the spacecraft dynamic model during the environmental qualification test, conducted on a large shaker table.
\end{abstract}

For any model correlation effort, the key has always been finding a proper definition of the boundary conditions. This paper is a correlation case study to investigate the difference in responses of a simple structure using a free-free boundary, a "fixed" boundary on the shaker table, and a base-drive vibration test, all using identical instrumentation. The NAVCON Jim Beam test structure, featured in the IMAC XXVII round robin modal test of 2009, was selected as a simple, well recognized and well characterized structure to conduct this investigation.

First, a free-free impact modal test of the Jim Beam was done as an experimental control. Second, the Jim Beam was mounted to a large 22,000 lbf shaker, and an impact modal test in this pseudo-fixed configuration was conducted. Lastly, a vibration test of the Jim Beam was conducted on the shaker table. The free-free impact test, the "fixed" impact test, and the base-drive test were used to assess the effect of the shaker modes, evaluate the validity of fixed-base modeling assumptions, and compare final model correlation results between these boundary conditions.

\section{Background and Motivation}

Launching a spacecraft into space is a costly endeavor. As a product of the current budget constraints, spacecraft programs are continually looking for ways to cut costs. There is a very tangible push to reduce expensive system level testing, and this has led many spacecraft programs to omit expensive full-scale modal tests from their schedule and rely solely on the system level environmental qualification test for model verification. This approach has two primary drawbacks [1]: 1. Data 
collected from a system level vibration test for correlation is limited compared to a full-scale modal test. 2. Spacecraft model correlation is not complete until very late in the launch campaign, which may pose a schedule risk to the program.

NASA's Launch Services Program (LSP) has minimum correlation criteria for spacecraft opting to correlate the spacecraft model during environmental qualification test. A Frequency Response Function (FRF) derived from a basedrive analysis of the spacecraft stack Finite Element Model (FEM) must envelope the levels observed during test; primary modes should match test frequencies within 5\%, and secondary modes should match within 10\% [1]. The analytical basedrive is typically conducted using the classic method of adding a rigid seismic mass to the base of the model and driving the system.

Inherently, this analysis fixes the base of the spacecraft and assumes that there is no flexibility in the table.

It has been this author's observation that it is difficult to achieve good correlation of modes beyond the first two bending modes and first axial mode. Further hampering model correlation efforts is the difference in accelerometer placement during an environmental qualification test from a modal test. For a qualification test, accelerometers are placed at a component's center of mass or its interface to ensure proper vibration levels are achieved, not necessarily at locations that would lend themselves to capturing frequencies and mode shapes for model validation. Model correlation is usually a secondary objective to the primary programmatic requirement of reaching the required qualification levels for the interface acceleration.

As stated by Mayes [3], there is a need for Finite Element validation using a fixed base condition, typical of payloads on a delivery system. This study investigates the difference in responses of a simple structure in a free-free boundary condition and "fixed" to a shaker table using identical instrumentation. Both tests are used to validate the FEM, and the results of each correlation are compared. Throughout this paper, the term "fixed" test will be used to refer to the impact modal test data taken of the Jim Beam while bolted to a stationary powered-off shaker table. The term "fixed" will remain in quotes, as this paper will demonstrate what the intuitive reader already knows: this boundary condition is not truly fixed.

\section{Can a Shaker Table Be Used to Correlate a FEM?}

There are many publications available which give insight into some of the challenges of correlating a FEM on a shaker table and some of the techniques employed to address this unique boundary condition; a few of them are highlighted here. Aerospace Corporation has several papers on the subject, but most notably has reported the bending mode frequency of a spacecraft was $25 \%$ lower than the frequency derived from modal test of the same spacecraft. The difference was traced to the flexibility of the table [6]. Sandia National Labs reported modes attributable to the base-shake table, which violates the assumed theoretical boundary condition of zero motion at the attachment point, except for the prescribed input [4]. This finding is of particular interest to this study, as it essentially invalidates the fixed base modeling assumption inherent in a seismic basedrive analysis. Sandia has since done additional work to identify the effects of the shaker table itself by using a separate small modal shaker to characterize the table and update the extracted FRFs and match modal mass [3]. This is likely one of the more robust ways to characterize the table, however, characterizing the table itself is beyond the scope of this paper, as typically very little characterization, if any, is done on shaker tables during spacecraft qualification testing.

\section{Meet Jim Beam}

The goal of this paper is to illustrate the effect of three separate boundary conditions on model correlation using a simple structure. The simple structure chosen for this investigation was the NAVCON Jim Beam, a simple three plate structure that was featured as part of the 2009 IMAC XXVII round-robin modal test. The Jim Beam has been tested well over 900 times by industry and academia, lending itself to being an ideal "experimental control". It is a simple, well recognized and well characterized structure to conduct this investigation. The Jim Beam is comprised of three aluminum plates and six screws, and weighs approximately $6.5 \mathrm{lbs}$ (Figure 1).

Modeling of the Jim Beam was done in FEMAP and analyzed using NX Nastran. Approximately 26,000 plate elements were used to model the three plates. This mesh size was driven by sizing studies conducted in support of modal testing done on 
each of the plates individually, and was required to capture the high frequency modes of the gold plate. The most sensitive part of the model was the joints. Trying to model the joint between the center gold plate and each of the other plates with only three bolt locations proved to be inadequate to capture the joint dynamics; instead, 11 "stiff" CBUSH spring-elements were used along the plate connections to simulate the joint compliance. For the free-free modal test model, four soft CBUSH spring-elements were used to simulate the bungees suspending Jim Beam (Figure 2).

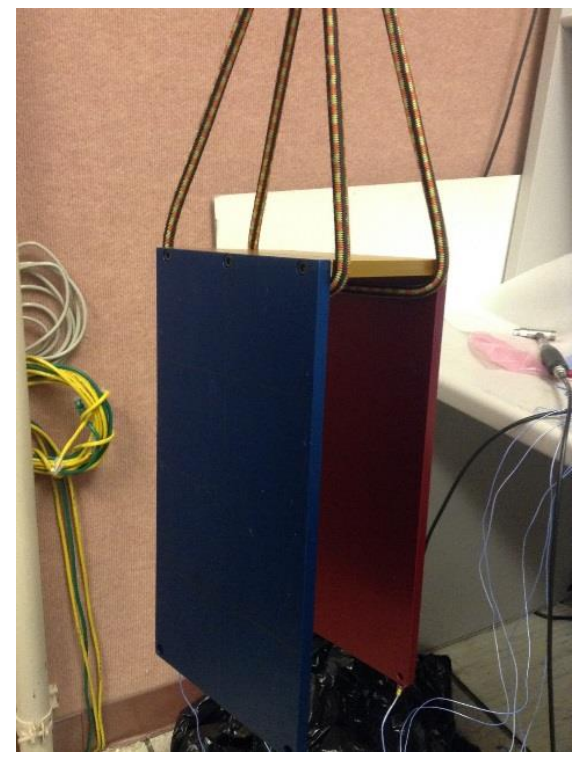

Figure 1 - Jim Beam Test Article

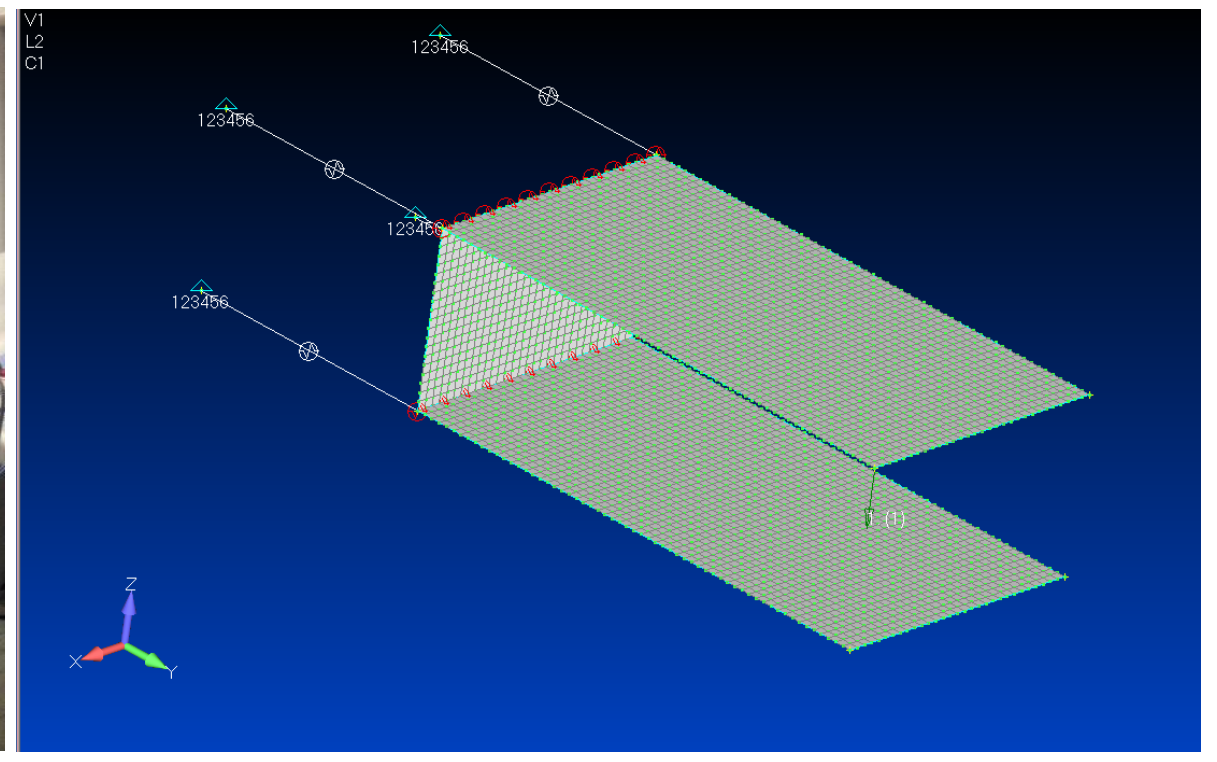

Figure 2 - Jim Beam Bungee Configuration Model

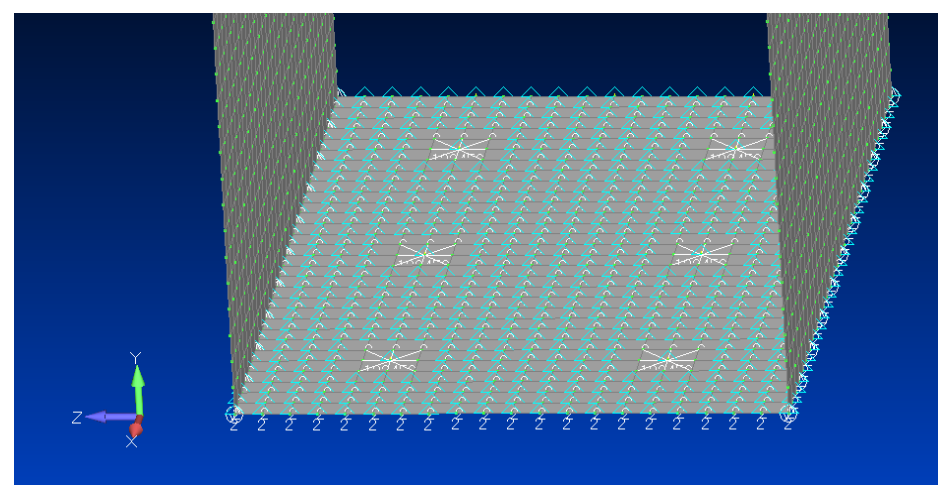

Figure 3 - Jim Beam Fixed Boundary

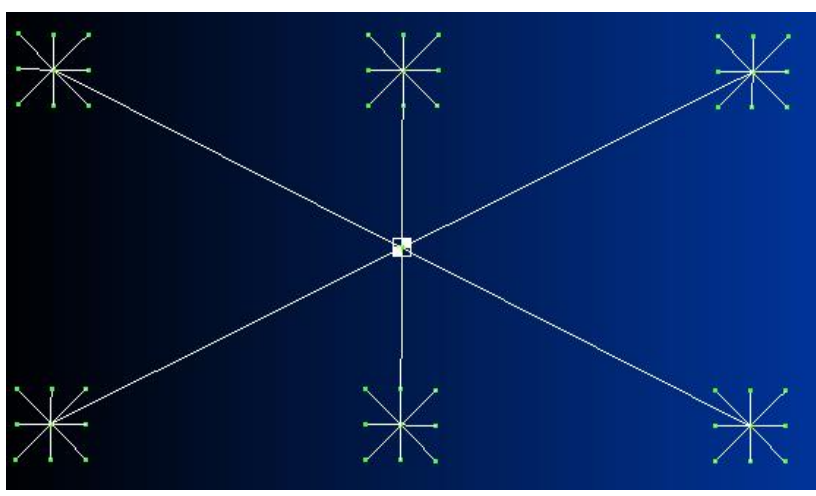

Figure 4 - Jim Beam Seismic Mass Boundary

From this point forward to avoid confusion, the six bolts connecting the three plates of the Jim Beam will be referred to as the connection bolts, while the six bolts at the interface between the Jim Beam and shaker table will be referred to as the interface bolts.

For the "fixed" base modal test, it was found that fixing only a single node at each of the six interface bolts was insufficient to capture the stiffness of the Jim Beam interface with the table. Therefore, the center node of each bolt was fixed in all six Degrees-Of-Freedom (DOF) using a Nastran SPC card, and rigid Nastran RBE2 "spiders" were used to connect that center node to the nine adjacent nodes surrounding it. Additionally, it was found this constraint alone still allowed too much compliance between the Jim Beam and the flat shaker table interface, as the physical test setup constrained the gold plate from bending. Therefore, in the analysis the entire gold plate was constrained in the Axial Y-direction (DOF 2) to better match mode shape animations (Figure 3). As noted previously, modeling the Jim Beam interface in this manner incorrectly assumes a fixed interface, as if the test article was mounted to a rigid modal plate. 
The model used for the "fixed" modal test was modified to simulate a seismic baseshake. The Y-direction constraint was removed, and a seismic mass (Nastran CONM2) was added to the center of the gold plate. The SPC constraint at the center node of each interface bolt was removed in lieu of an additional rigid RBE2 "spider" connecting the center of each bolt to the seismic mass (Figure 4).

\section{The Free-Free Test}

The first boundary condition investigated was the classic free-free condition, where the Jim Beam was hung by bungees and modal impact data was taken (Figure 1). One of the focal points of the original Jim Beam round-robin testing was varying torque from 30 in-lbs to 60 in-lbs on the six connection bolts between the three plates and observing the effect on the modal test results. As the effect of bolt torque was not a focus of this paper, the torques of these six connection bolts was kept constant at $60 \mathrm{in}-\mathrm{lbs}$, and verified before each of the three different tests were conducted. Results of the Jim Beam free-free modal test were compared with free-free results published by ATA [5] as a "truth test" to verify the test methodology and data processing. Results are compared below in Table 1:

\begin{tabular}{|r|r|r|r|}
\hline \multicolumn{3}{|c|}{ Free-Free Modal Test Frequency Comparison } \\
\hline Mode & \multicolumn{1}{|c|}{ ATA $^{[5]}$} & \multicolumn{1}{|c|}{ KSC } & \% Difference \\
\hline 1 & 59 & 64 & 8.47 \\
2 & 159 & 164 & 3.14 \\
3 & 209 & 224 & 7.18 \\
4 & 346 & 352 & 1.73 \\
5 & 446 & 460 & 3.14 \\
6 & 494 & 505 & 2.23 \\
7 & 601 & 635 & 5.66 \\
8 & 1126 & 1144 & 1.60 \\
9 & 1216 & 1244 & 2.30 \\
10 & 1332 & 1348 & 1.20 \\
\hline
\end{tabular}

Table 1 - Frequency Comparison ATA vs KSC

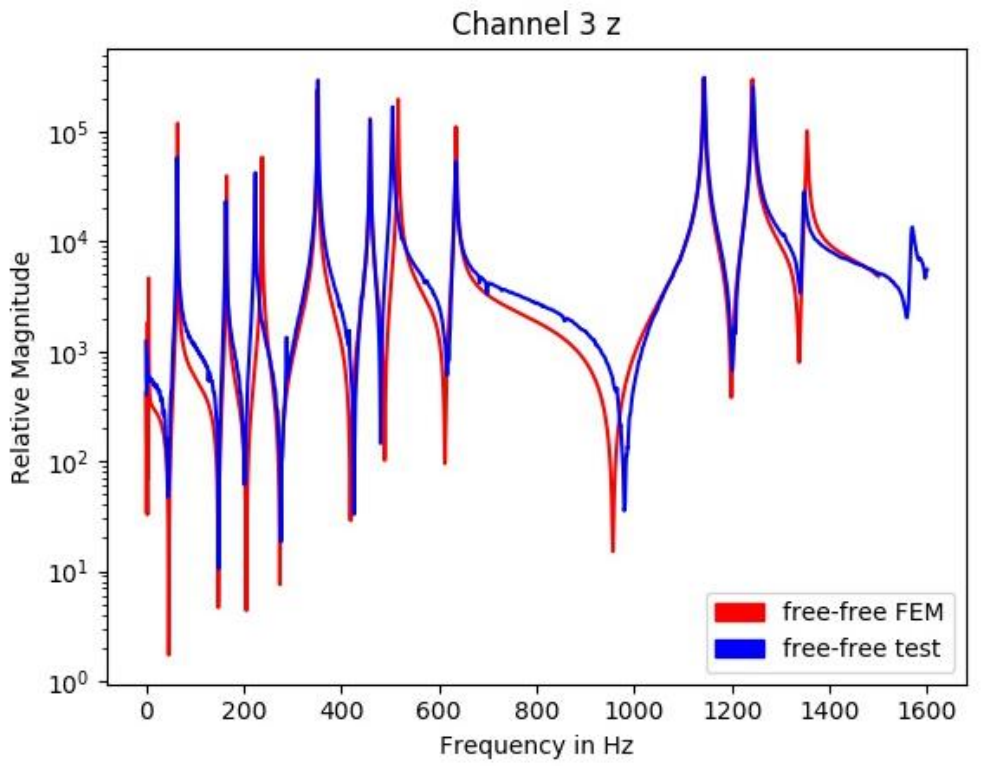

Figure 5 - FRF of Free-Free Test vs FEM

Overall, the frequency comparison between ATA and KSC is rather good considering the variability of lab equipment, analysts, and bungeed boundary conditions. The first mode shows the largest difference, likely due to slight differences in how KSC and ATA suspended the Jim Beam, and Mode 3 was a torsion mode that was difficult to excite and capture with impact modal testing. Having verified the test methodology, the FEM was correlated to the KSC test data to act as the baseline verified model for the "fixed" test and shaker test. NX Nastran Solution 111 was used to synthesize modal hammer impulse excitations, and post processing of the results was completed using Python software to generate FRF comparisons. A FRF comparison between the modal test and the correlated FEM are given in Figure 5. Channel 3Z, located at the tip of the red plate, was chosen for test vs FEM comparisons, as this single channel captured the majority of clean system modes.

\section{The "Fixed" Test}

Following completion of the free-free test, the Jim Beam was modified for vibration table fixturing. Six half-inch holes with two-inch spacing were drilled into the gold plate of the Jim Beam (Figure 6) to interface with an existing 1x1x1 foot vibe table adaptor Cube, weighing approximately 107 lbs. The Cube was mounted to an Unholtz-Dickie T1000 22,000 lbf shaker. The same data acquisition system, computer, accelerometers, accelerometer locations and impact points as the free-free test were used, with the exception of nine impact points on the bottom of the gold plate that were inaccessible due to the new mounting configuration. 


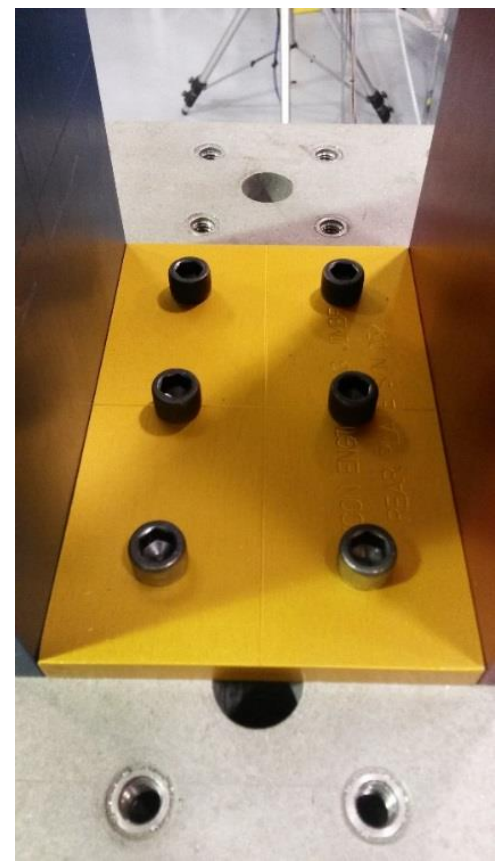

Figure 6 - Jim Beam Six Interface Bolts

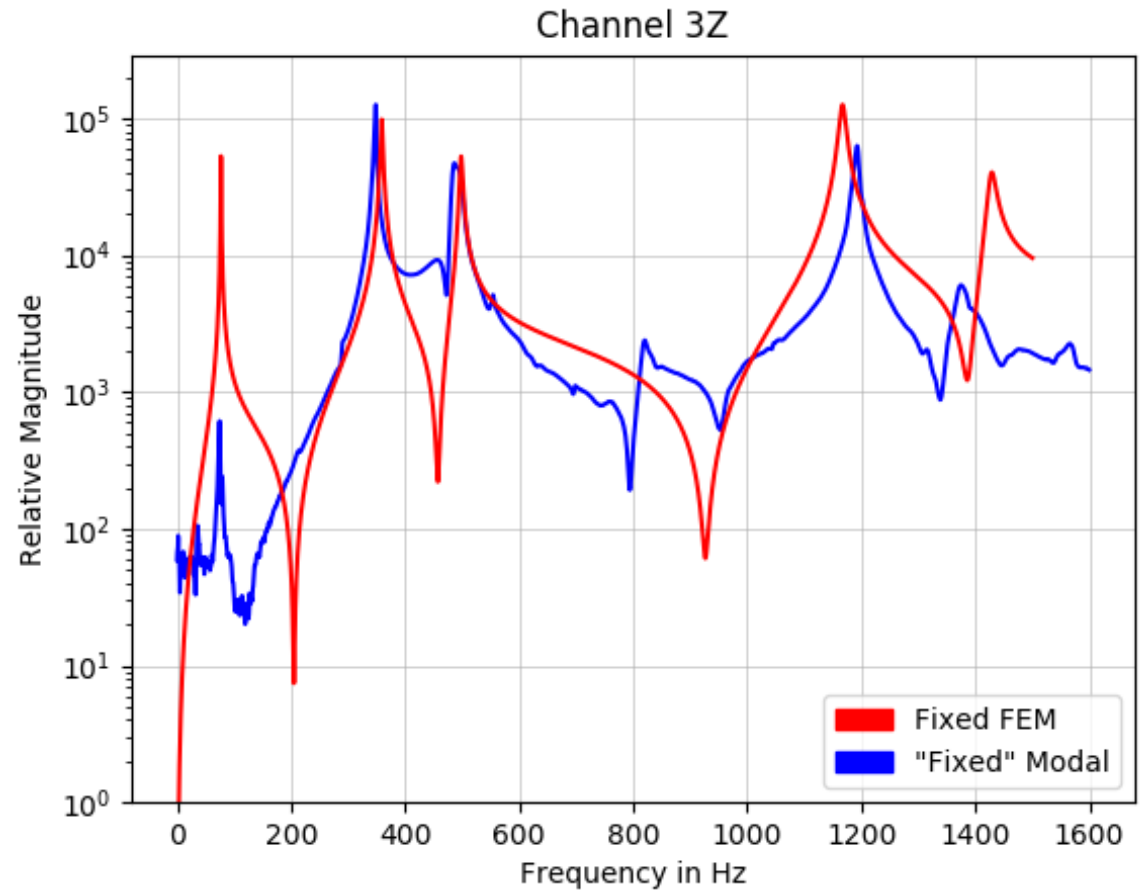

Figure 7 - FRF of "Fixed" Modal Test vs. FEM

As with the free-free test, NX Nastran Solution 111 was used to synthesize modal hammer impulse excitations, and post processing of the results was completed using Python software. Channel 3 was once again used for FRF comparisons between FEM and test (Figure 7). Observing differences between the free-free and "fixed" base plots (Figures 5 and 7) immediately reveals that while the "fixed" base FEM follows the test response, the comparison is not nearly as good as the correlation achieved with the free-free boundary condition. It was quite difficult to obtain clean modal impact data and mode shapes mounted to the shaker table, as impacts on the blue plate could not transfer enough energy through the bolted interface to excite the red plate, and vice versa. If the vibe table were a perfect fixed interface as it was modeled, there would be zero motion in the red plate when the blue plate was excited. The fact that any motion at all was captured in the red plate during the modal test is indicative of an imperfect fixation. It is also important to note that the table was powered off for the "fixed" test, which means the air bearings within the vibe table were not active. Practically, this means that there was some undetermined compliance in the shaker head/armature. A good shove on the interface Cube could induce noticeable oscillation of the Cube with the attached Jim Beam. This is likely why the first mode was difficult to resolve during the "fixed" modal test, and why a model correlated in the free-free realm was no longer correlated on this quasi-fixed shaker boundary condition.

\section{The Sine Vibration Test}

Once the "fixed" modal test was complete, the shaker was powered on for the sine vibration test. For this test, the KSC vibration lab's 10 channel data acquisition system was used, as it interfaces with the shaker table control system. The same five teardrop accelerometers on the Jim Beam were kept at the same locations as for the previous two modal tests, and five additional single-axis accelerometers were added to the Cube; one close to the Jim Beam interface to be used as the control channel, and one at each of the four corners of the Cube. This was to capture any rotations present in the Cube, especially during the lateral runs where the Jim Beam was cantilevered off the side of the Cube (Figure 8).

Sine sweeps were run in all three axes (XYZ) from 5-3000 Hz at quarter-G and half-G levels, sweeping at two octaves/min. The Jim Beam was rotated on the Cube after each test to drive the next test axis. No frequency shifts were observed between the quarter-G and half-G testing, as the Jim Beam is a very simple and linear structure. 
The effect of directionality on the fixed-base modes of the Sine Vibe results is clear from Figure 9 - all three test axes are required to capture all the fixed base modes. By contrast, a single reference accelerometer could be used to capture all of the free-free modes.
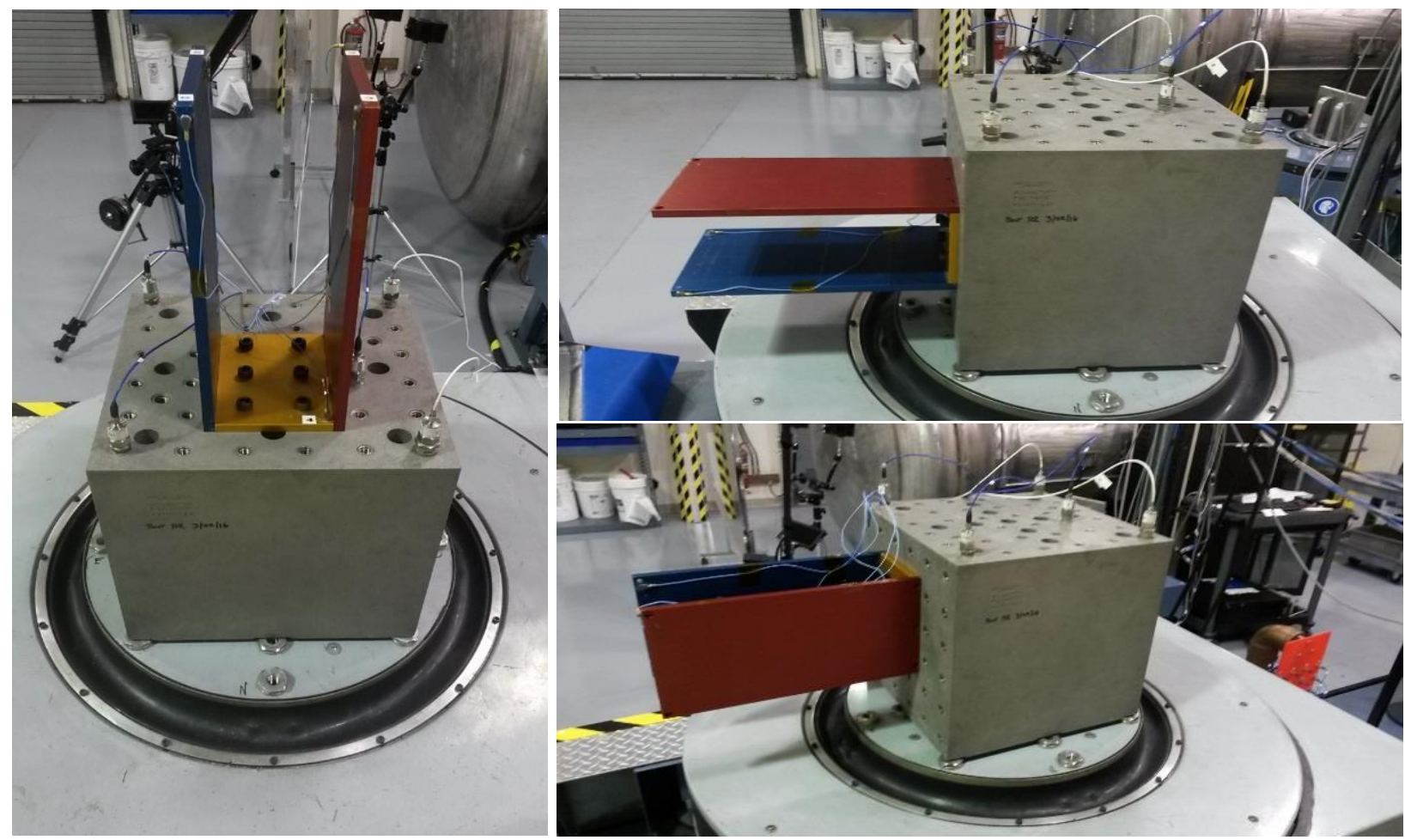

Figure 8 - Jim Beam Vibe Table Test Configurations. Clockwise from Bottom Right: X-Axis, Y-Axis, Z-Axis

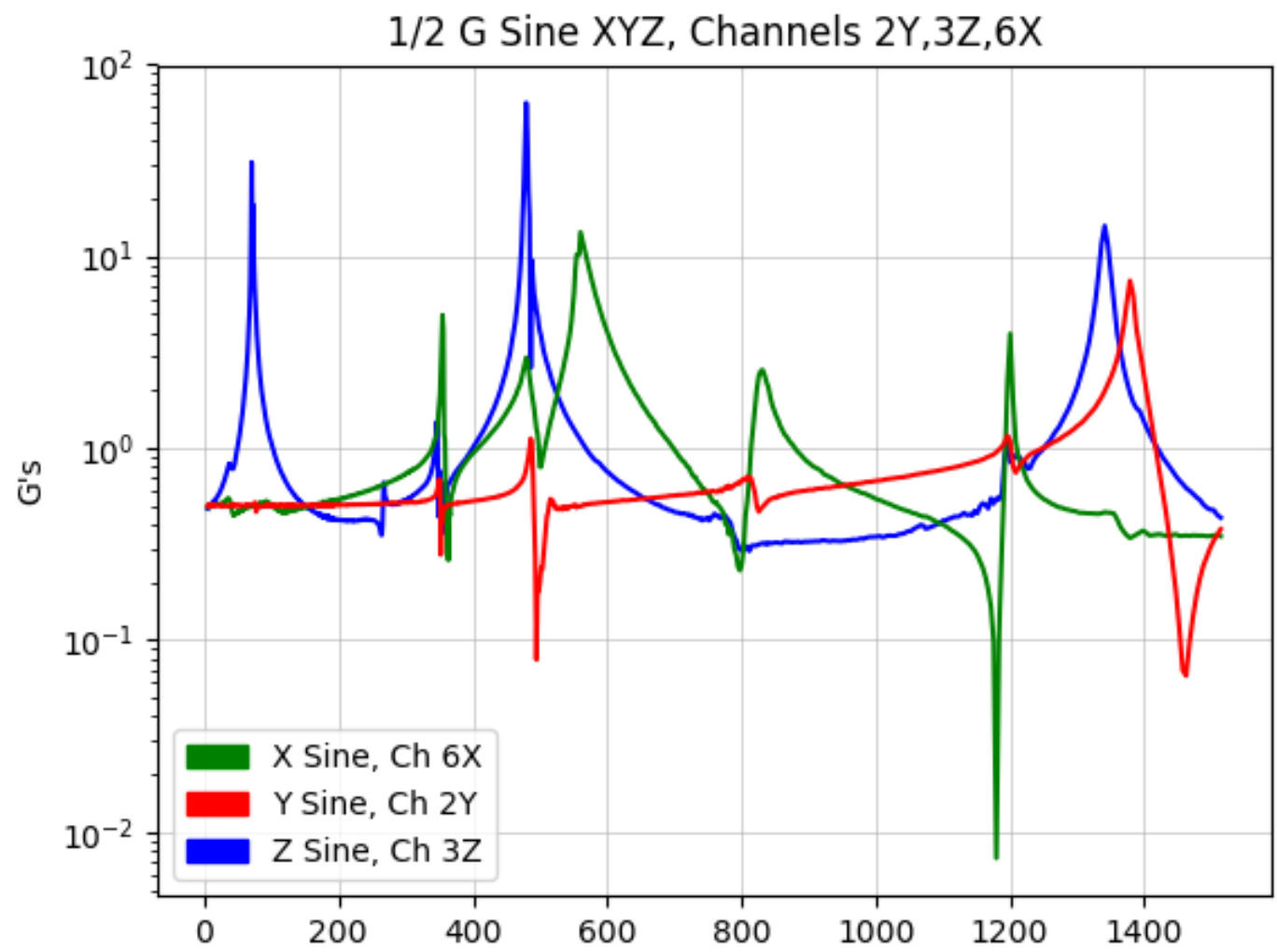

Figure 9 - Sine Vibration Test, Channels 6X, 2 Y, and $3 Z$ 
NX Nastran Solution 111 was used to synthesize seismic basedrive responses, and post processing of the results was completed using Python software to generate Frequency-Response-Function (FRF) comparisons, given in Figure 10.
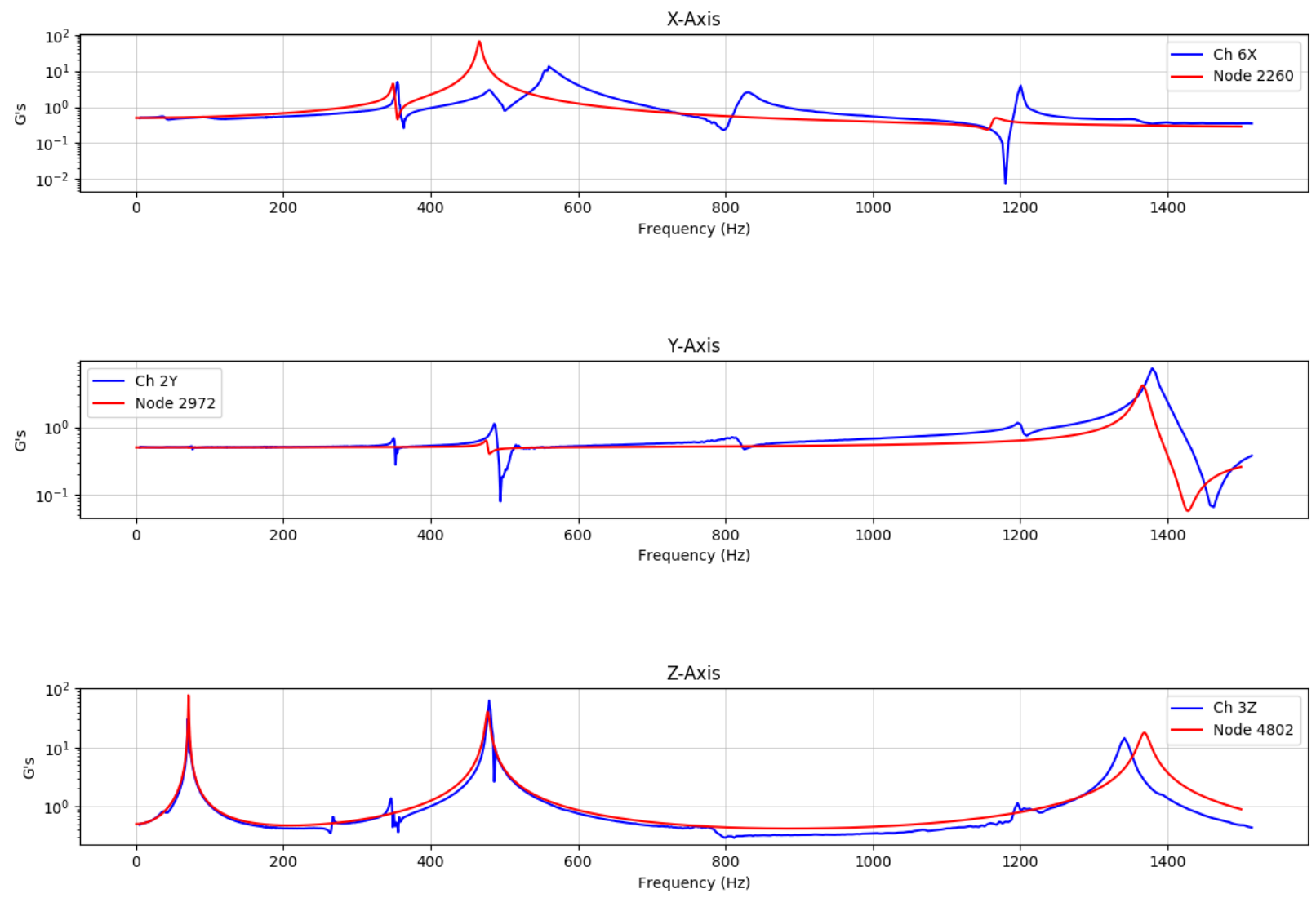

Figure 10 - Comparison of each test axis with its respective accelerometer

It is clear from the data that the sine vibration test is picking up something that the analytical basedrive did not. In particular, the X-Axis test data does not show clean modes between 400 and $600 \mathrm{~Hz}$, and an additional mode appears around $800 \mathrm{~Hz}$ in the X-Axis that wasn't seen during the free-free test. One might surmise that these effects are due to the cantilevered test configuration, however, upon further inspection, the $800 \mathrm{~Hz}$ mode can also be seen in the "fixed" modal test data, which was taken while the Jim Beam was bolted upright in the Y-Axis. Additionally, the analysis shifts the high frequency modes above $1100 \mathrm{~Hz}$. Given that the analysis was conducted using a correlated FEM, the only new element is the unknown compliance of the shaker table. However, without an a-priori correlation, and just the sine vibration data, would this model be considered uncorrelated? Would time and effort be spent changing the model to match shaker table effects, which do not actually reflect the test article or the flight interface?

\section{Conclusions}

The ability to capture the boundary conditions of the test in the model dictate the success of the correlation. Thus most modal tests control the boundary by using a modal plate to assume a fixed boundary condition or very soft springs such as bungees or airbags to assume a free-free boundary condition. The difficulty in using a sine vibration test for model correlation is the definition of the shaker boundary, as there is compliance in the system that cannot be accounted for in the model with a classic seismic basedrive, which is an industry standard practice. The results presented here validate the supposition of Carne [4] that the shaker table cannot be assumed to have zero motion at the interface point, as a seismic basedrive implies. In this study, it was easy to model bungees as light springs and produce excellent correlation results, where most of the time and effort spent on model correlation was devoted to updating the Jim Beam FEM. To achieve similar correlation results with the sine vibration test, most time and effort would be spent accounting for the shaker 
boundary condition, not the Jim Beam model itself. In this case, the sine vibration test was completed on a correlated Jim Beam FEM, highlighting the poor definition of the shaker boundary. The luxury of a correlated FEM going into sine vibration testing is not afforded to most spacecraft, as the vibration test may be the only opportunity to obtain empirical data for model correlation.

\section{Acknowledgements}

The authors would like to thank NAVCON Engineering for providing a Jim Beam for study, and KSC Vibrations Lab Test Director Mark Hamilton for time in the vibration lab.

\section{Bibliography}

1. Abdallah, Ayman. Verification of Spacecraft Dynamic Models for Coupled Loads Analysis. Kennedy Space Center, Florida: NASA, Nov. 2007

2. Avitabile, Pete. "Why can't I Run a Modal Test With One Big Shaker and Just 'Crank Up the Signal'?" Modal Space- In Our Own Little World 83. University of Massachusetts Lowell, Apr. 2011

3. Bridgers, Daniel L., and Randy L. Mayes. Extracting Fixed Base Modal Models from Vibration Tests on Flexible Tables. Albequerque, New Mexico: Sandia National Laboratories, Oct. 2009

4. Carne, Thomas G., Martinez, David R., Nord, Arlo R. A Comparison of Fixed-Base and Driven-Base Modal Testing of an Electronics Package. Albuquerque, New Mexico: Sandia National Laboratories, 1989

5. Hoople, Gordon. Jim Beam Testing \& Results. IMAC XXVII. Orlando, Florida, 2009.

6. Lollock, Jeffrey A. A Comparison of Base-shake and Mode Survey Test Based Model Correlation Techniques. Los Angeles, California: The Aerospace Corporation, 2004 